\title{
Article
}

\section{Synergistic Effects of Venetoclax and Daratumumab on Antibody-Dependent Cell-Mediated Natural Killer Cytotoxicity in Multiple Myeloma}

\author{
Ayano Nakamura ${ }^{1}$, Susumu Suzuki ${ }^{2,3, *}$, Jo Kanasugi ${ }^{1}$, Masayuki Ejiri ${ }^{4}$, Ichiro Hanamura ${ }^{1}{ }^{\oplus}$, Ryuzo Ueda ${ }^{3}$, \\ Masao Seto ${ }^{1}$ and Akiyoshi Takami ${ }^{1}$ D \\ 1 Department of Internal Medicine, Division of Hematology, Aichi Medical University School of Medicine, \\ Nagakute 480-1195, Japan; ayano.n@aichi-med-u.ac.jp (A.N.); jo@aichi-med-u.ac.jp (J.K.); \\ hanamura@aichi-med-u.ac.jp (I.H.); seto_masao@aichi-med-u.ac.jp (M.S.); takami-knz@umin.ac.jp (A.T.) \\ 2 Research Creation Support Center, Aichi Medical University, Nagakute 480-1195, Japan \\ 3 Department of Tumor Immunology, Aichi Medical University School of Medicine, Nagakute 480-1195, Japan; \\ uedaryu@aichi-med-u.ac.jp \\ 4 Department of Pharmacy, University Hospital, Aichi Medical University, Nagakute 480-1195, Japan; \\ ejiri.masayuki.107@mail.aichi-med-u.ac.jp \\ * Correspondence: suzukis@aichi-med-u.ac.jp; Tel.: +81-561-62-3311 (ext. 11426)
}

\section{check for}

updates

Citation: Nakamura, A.; Suzuki, S.; Kanasugi, J.; Ejiri, M.; Hanamura, I.; Ueda, R.; Seto, M.; Takami, A. Synergistic Effects of Venetoclax and Daratumumab on

Antibody-Dependent Cell-Mediated Natural Killer Cytotoxicity in Multiple Myeloma. Int. J. Mol. Sci. 2021, 22, 10761. https://doi.org/ 10.3390/ijms221910761

Academic Editor: Lina Ghibelli

Received: 3 June 2021

Accepted: 1 October 2021

Published: 5 October 2021

Publisher's Note: MDPI stays neutral with regard to jurisdictional claims in published maps and institutional affiliations.

Copyright: (c) 2021 by the authors. Licensee MDPI, Basel, Switzerland. This article is an open access article distributed under the terms and conditions of the Creative Commons Attribution (CC BY) license (https:// creativecommons.org/licenses/by/ $4.0 /)$.

\begin{abstract}
The prognosis of multiple myeloma (MM) has drastically improved owing to the development of new drugs, such as proteasome inhibitors and immunomodulatory drugs. Nevertheless, MM is an extremely challenging disease, and many patients are still refractory to the existing therapies, thus requiring new treatment alternatives. Venetoclax is a selective, orally bioavailable inhibitor of BCL-2 that shows efficacy in MM not only as a single agent but also in combination therapy, especially for $\mathrm{MM}$ patients with translocation $\mathrm{t}(11 ; 14)$. However, many patients are refractory to this drug. Here, we treated the MM cell lines KMS12PE and KMS27 with a combination treatment of venetoclax targeting BCL-2 and daratumumab targeting CD38 to evaluate the synergistic cytotoxicity of these drugs in vitro. MM cell lines were co-cultured with natural killer (NK) cells at an effector:target ratio of 0.3:1 in the presence of serial concentrations of daratumumab and venetoclax, and the resulting apoptotic MM cells were detected by flow cytometry using annexin V. These results indicated that the antibody-dependent cell-mediated NK cytotoxicity was enhanced in KMS12PE and KMS27 cells harboring $\mathrm{t}(11 ; 14)$ with a high BCL-2 expression, suggesting that the combination treatment of venetoclax and daratumumab should be especially effective in patients with these characteristics.
\end{abstract}

Keywords: multiple myeloma; daratumumab; venetoclax; BCL-2; ADCC; NK cell

\section{Introduction}

The clinical introduction of proteasome inhibitors (PIs), such as bortezomib, and immunomodulatory drugs (IMiDs), such as thalidomide and lenalidomide, significantly improved the prognosis for multiple myeloma (MM) patients. Treatment with monoclonal antibodies has been shown to be effective for MM patients refractory to PIs and IMiDs, which was demonstrated by daratumumab targeting CD38 and elotuzumab targeting SLAMF7. Although many patients are currently successfully treated and achieve a complete response, many experience relapse in the long term and require additional treatment options [1].

The BCL-2 family of proteins is composed of crucial apoptosis regulators [2]. These include pro-survival and pro-apoptotic proteins, and interactions between these two classes of proteins are crucial in making cell fate decisions [2]. BCL-2 proteins are classified into three major groups according to structure and function: anti-apoptosis proteins include MCL-1, BCL-2, and BCL-XL; multi-domain pro-apoptotic proteins include BAX and BAK1; and $\mathrm{BH} 3$-only proteins include BIM, BID, BAD, and NOXA [2]. 
Anti-apoptosis proteins are important therapeutic targets [2] and are targeted by venetoclax (also known as ABT-199), a selective, orally bioavailable and BH3 mimetic inhibitor with high affinity to BCL-2 but not to BCL-XL or MCL-1 [2]. Venetoclax is regarded as a new option for the treatment of $\mathrm{MM}$, with a mechanism of action different from that of PIs and IMiDs; it is currently being evaluated through clinical trials in patients with MM [2]. Venetoclax induced apoptosis in human MM cell lines and in vitro primary samples collected from patients with MM, especially in those harboring translocation $\mathrm{t}(11: 14)$ (q13:q32) [3,4], which is present in $15 \%$ to $20 \%$ of patients with MM [3,4].

In the phase I study of venetoclax monotherapy for relapsed/refractory $(R / R) M M$, the overall response rate (ORR) was $21 \%$, and $15 \%$ of patients achieved a very good response rate (VGPR) or better (NCT01794520) [5]. Within the group of patients carrying $t(11 ; 14)$, the ORR was $40 \%$, and $27 \%$ patients achieved a VGPR or better [5]. In this study, patients with high gene expression ratios of $B C L 2$ to $B C L 2 L 1$ and $B C L 2$ to $M C L 1$ were more sensitive to venetoclax than patients with low ratios [5].

Venetoclax has shown efficacy for MM not only in monotherapy but also in combination therapy. In a phase $\mathrm{Ib}$ study evaluating venetoclax together with bortezomib and dexamethasone in R/R MM, the ORR was $67 \%$, and $42 \%$ of patients achieved a VGPR or better (NCT01794507) [6]. In this study, 94\% of the patients with high BCL2 levels achieved ORR, while in those with low BCL2 levels, the ORR was 59\%. Bortezomib inhibits MCL-1 indirectly by stabilizing the MCL-1-neutralizing protein NOXA [7]. In xenograft models resistant to venetoclax that co-express BCL-XL or MCL-1 with BCL-2, this resistance was decreased by bortezomib [8]. Similarly, dexamethasone upregulates the pro-apoptotic $\mathrm{BIM}$ and increases its binding to BCL-2, which also results in increased sensitivity to venetoclax $[6,9]$.

Some patients do not respond to this drug, while others show progress after an initial response. The efficacy and safety of a treatment using venetoclax together with daratumumab and dexamethasone (VenDd) is currently being evaluated (NCT03314181) [2]. Bortezomib and dexamethasone show similarities to venetoclax in that they target the BCL-2 family and improve treatment effectivity. The antibody-dependent cell-mediated cytotoxicity (ADCC) apoptotic pathway also activates BID, a BH3-only protein from the BCL-2 family, to induce BAX activation. In an attempt to explore a new approach for the treatment of patients with $\mathrm{MM}$, here we aimed to evaluate, using in vitro techniques, whether the combination treatment of venetoclax and daratumumab increased cytotoxicity. Our results will contribute to future studies on the clinical applications of this type of treatment, including the establishment of the optimal dose for each of these drugs.

\section{Results}

2.1. Expression Levels of CD38 in MM Cell Lines

CD38 was highly expressed in KMS12PE and moderately expressed in KMS27 and KM5; in contrast, CD38 expression was low in U266 (Figure 1). 

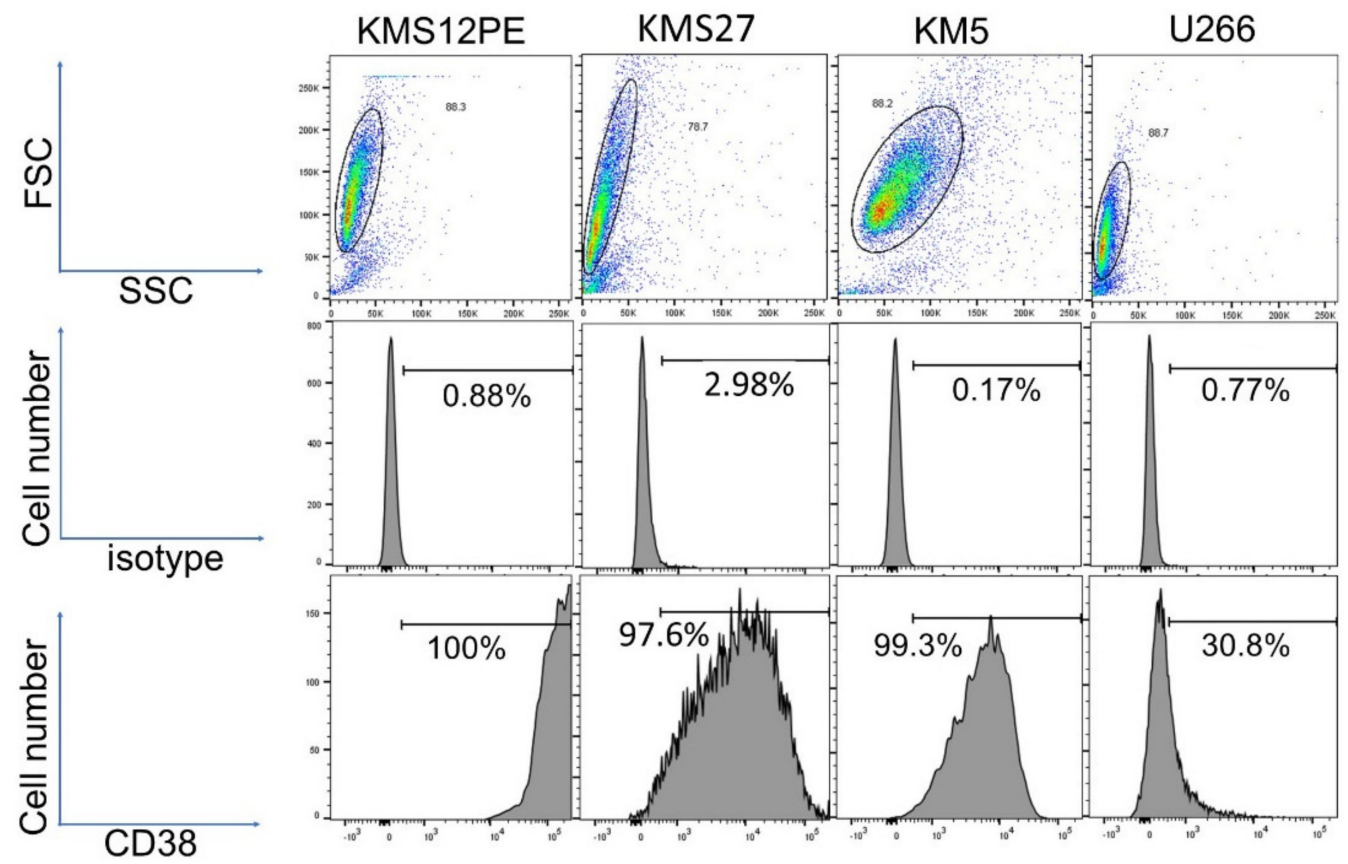

Figure 1. Flow cytometry for CD38 in MM cell lines. The upper panels show cytograms; the x-axis represents SSC and the y-axis represents FSC. The circled areas represent viable cells. The middle and lower panels show flow cytometry histograms. The x-axis shows the positivity for the antibodies and the $y$-axis represents the cell numbers. The middle panels indicate results with isotype control. The lower panels indicate results with Alexa 647-labeled CD38 antibody.

\subsection{Expression Levels of BCL-2 Family Proteins in MM Cell Lines}

BCL-2 family proteins levels varied among the MM cell lines (Figure 2). BCL-2 was more highly expressed in KMS12PE than in KMS27, U266, and KM5. High protein level of BCL-XL was observed in U266; in contrast, BCL-XL protein level was very low in KMS12PE, KMS27, and KM5. As for MCL-1, the protein level was higher in KM5 than in KMS12PE, KMS27, and U266. BCL-2:BCL-XL and BCL-2:MCL-1 ratios were higher in KMS12PE and KMS27 than in U266 and KM5. This observation correspondent to venetoclax sensitivity (Figure 3).

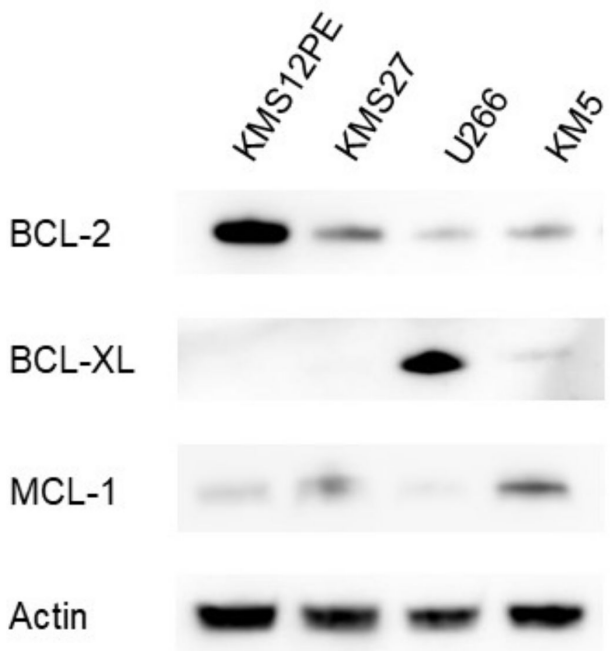

Figure 2. Western blot analyses for BCL-2 family proteins in MM cell lines. Actin was used as a loading control. 


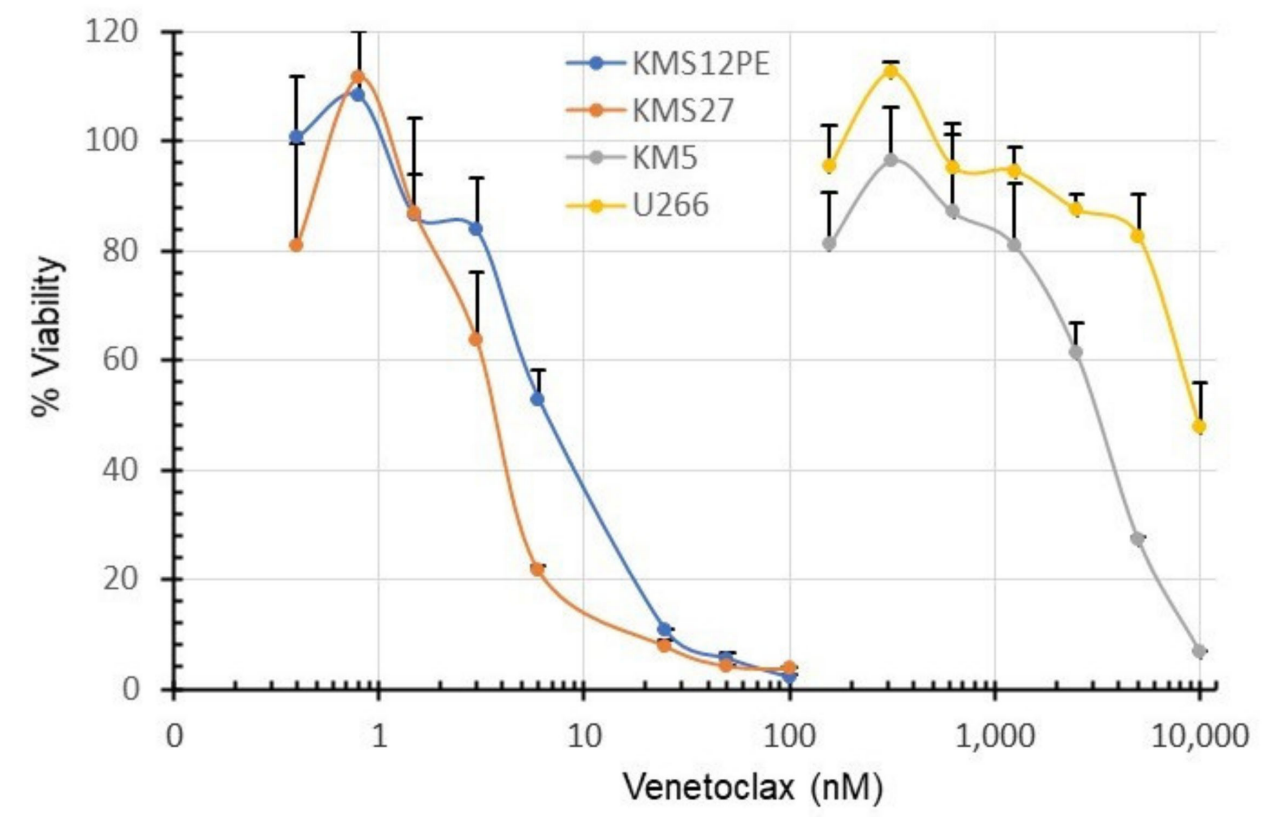

Figure 3. Cytotoxicity of venetoclax to MM cell lines. MM cell lines were treated with venetoclax at the indicated concentrations and cultured for $48 \mathrm{~h}$. The x-axis represents the concentration of venetoclax. The $\mathrm{x}$-axis was formatted to have a base 10 logarithmic scale. The $\mathrm{y}$-axis shows $\%$ viability. Viability values on each point is shown as $100 \%$ at $0 \mathrm{nM}$.

\subsection{Sensitivity of MM Cell Lines to Venetoclax}

The sensitivity of MM cell lines to venetoclax was examined by a WST-1 assay. As shown in Figure 3, the IC50 values of KMS12PE, KMS27, U266, and KM5 for venetoclax were 6.3, 3.5, 10000, and $3100 \mathrm{nM}$, respectively. KMS12PE and KMS27 were sensitive to venetoclax but KM5 and U266 were resistant.

\subsection{Synergistic Effects of Venetoclax and Daratumumab on ADCC}

In order to compare the cytotoxic activity of venetoclax alone, daratumumab alone, and a combination of both agents, flow cytometry was performed for detecting annexin V. Representative results are shown in Figure 4. In KMS12PE, the annexin V positivity was $19.3 \%$ when $2.5 \mathrm{nM}$ venetoclax was used alone, $41.9 \%$ when $25 \mathrm{ng} / \mathrm{mL}$ daratumumab was used alone, and $64.2 \%$ when a combination of $2.5 \mathrm{nM}$ venetoclax and $25 \mathrm{ng} / \mathrm{mL}$ daratumumab was used (Figure 4A). On the other hand, in KM5, the annexin V positivity was $15.1 \%$ when $2.5 \mu \mathrm{M}$ venetoclax was used alone, $40.6 \%$ when $25 \mathrm{ng} / \mathrm{mL}$ daratumumab was used alone, and $31.8 \%$ when a combination of $2.5 \mu \mathrm{M}$ venetoclax and $25 \mathrm{ng} / \mathrm{mL}$ daratumumab was used (Figure 4B). 
A

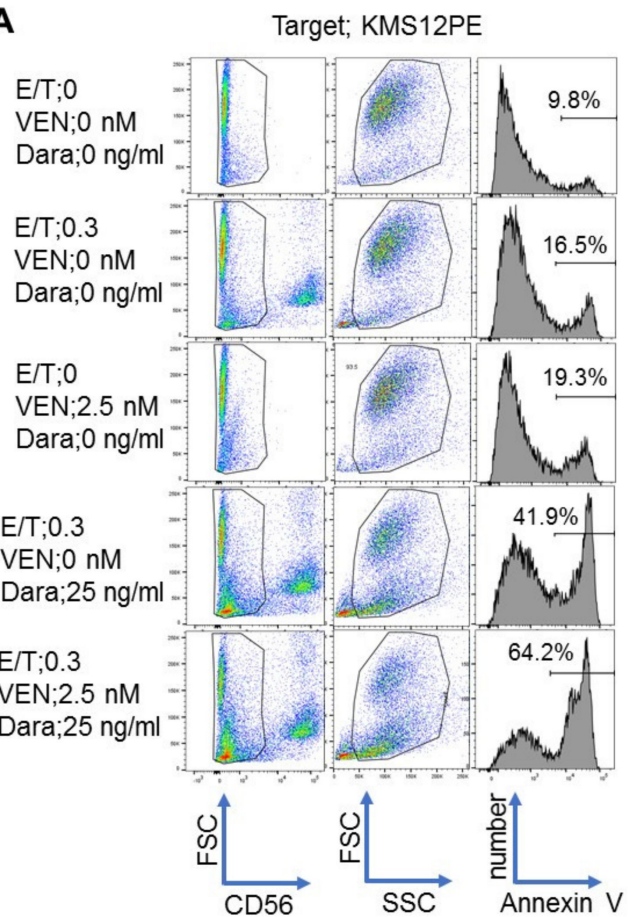

B

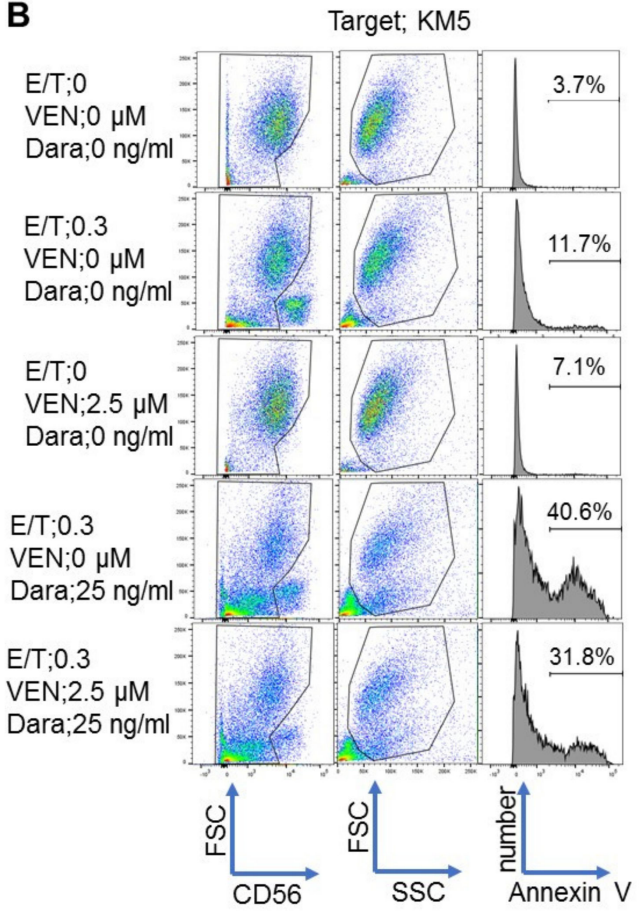

Figure 4. Representative flow cytometric analyses of cytotoxicity by NK cell- mediated ADCC with daratumumab and venetoclax to in MM cell lines using annexin V. The cytotoxicity of combining NK cell mediated ADCC with daratumumab and VEN to in KMS12PE (A) and KM5 (B) cells was investigated by flow cytometric analyses with annexin V. Cells were gated on forward scatter (FSC) and CD56 to remove NK cells, and the gated cells were separated by forward scatter (FSC) and side scatter (SSC). Annexin V+ cells in gated cells separated by FSC and SSC were detected. The percentage of Annexin V+ cells is indicated in each histogram. Cell treatment conditions are indicated on the left side of each panel. E/T: effector/target, VEN: venetoclax, Dara: daratumumab.

These experiments were repeated three times in each cell line, and the average value with standard deviation (SD) has been shown (Figure 5). A synergistic effect was observed in KMS12PE and KMS27, which are sensitive to venetoclax, but not in U266 and KM5, which are resistant to venetoclax. 


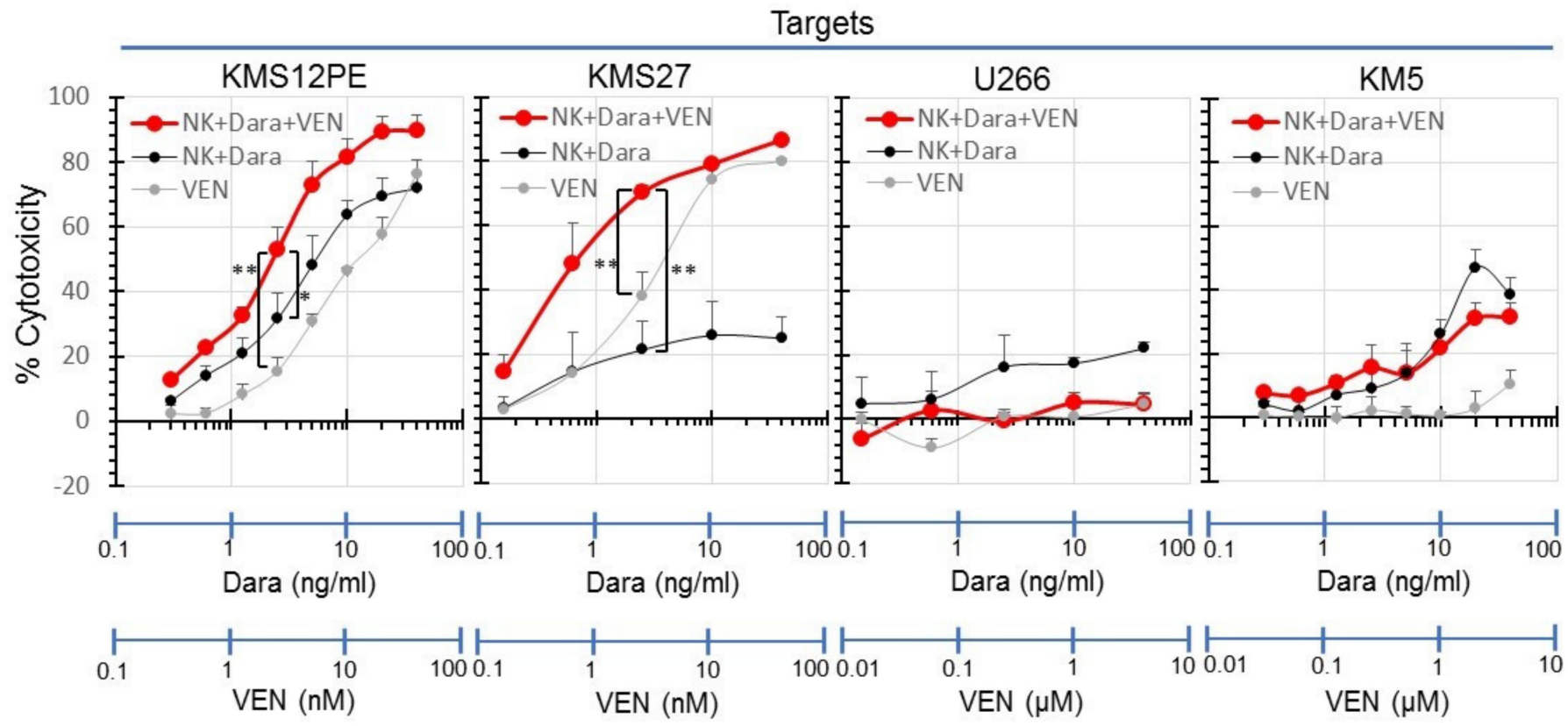

Figure 5. Synergistic cytotoxicity of NK cell mediated ADCC with daratumumab and venetoclax to MM cell lines. Cytotoxicity of NK cell mediated ADCC with daratumumab and venetoclax to MM cell lines, KMS12PE, KMS27, U266 and KM5 was measured by flow cytometry with annexin V. MM cell lines were cocultured with NK cells at effector/target $(\mathrm{E} / \mathrm{T})$ ratios: 0.3 with serial concentrations of daratumumab and venetoclax. Cytotoxicity is shown as the mean of three different assays. Red, black, and gray lines indicate the cytotoxicity of NK cells with daratumumab and venetoclax $(\mathrm{NK}+$ Dara + VEN), NK cells with daratumumab (NK + Dara), and venetoclax (VEN), respectively. * - differences between groups $0.01<p<0.05$, the Student's t-test. ${ }^{* *}$ - differences between groups $p<0.01$, the Student's t-test.

The perforin inhibitor concanamycin A (CMA) did not inhibit the cytotoxicity of venetoclax alone but clearly inhibited the synergistic cytotoxicity of daratumumab and venetoclax (Figure 6).

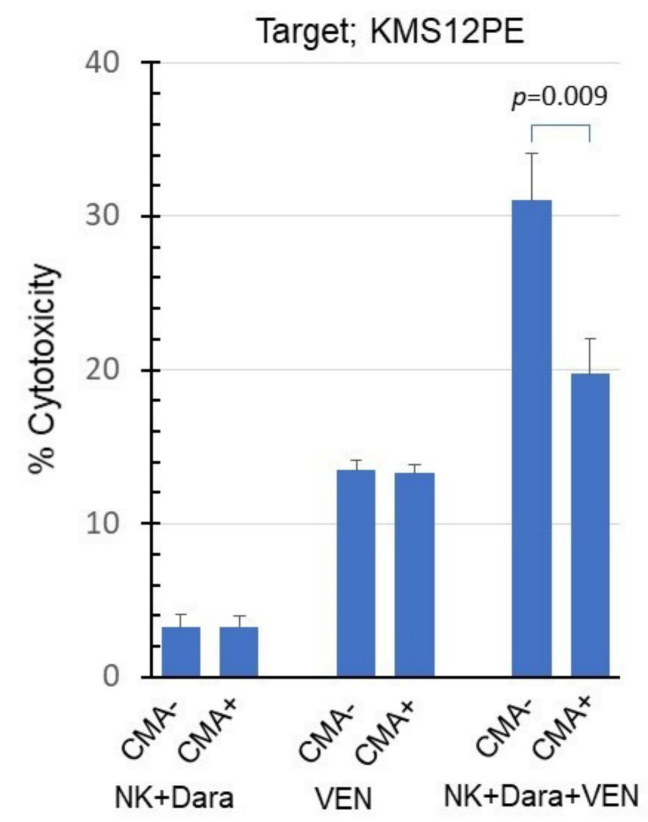

Figure 6. Inhibition of the synergistic cytotoxicity of daratumumab and venetoclax by CMA. KMS12PE was co-cultured with NK cells $(\mathrm{E} / \mathrm{T}=0.3)$, daratumumab $(2.5 \mathrm{ng} / \mathrm{mL})$, and venetoclax $(2.5 \mathrm{nM})$ in the presence of CMA $(10 \mathrm{nM})$ for $18 \mathrm{~h}$. 


\section{Discussion}

This study showed that venetoclax enhanced the ADCC induced by daratumumab in the MM cell lines that are sensitive to venetoclax. This was especially true for cell lines harboring $\mathrm{t}(11 ; 14)$ with high levels of CD38 and BCL-2. KMS12PE [10] and KMS27 [11] are examples of MM cell lines harboring $\mathrm{t}(11 ; 14)$ (q13:q32) and a high expression of BCL-2 [10]. The in vitro venetoclax monotherapy was more effective for MM in cell lines with $t(11 ; 14)$ than in those without $t(11 ; 14)$. However, U266 expressed low levels of BCL-2, despite the presence of $\mathrm{t}(11 ; 14)$ and resistance to venetoclax. The $\mathrm{t}(11 ; 14)$ translocation is a hallmark of mantle cell lymphoma and results in an overexpression of cyclin D1 [12]. The associations between $t(11 ; 14)$ and BCL-2 expression remain poorly understood. A phase I trial for patients with $\mathrm{R} / \mathrm{R} \mathrm{MM}$ revealed the effectiveness of venetoclax in the patients with high $B C L 2: B C L 2 L 1$ or $B C L 2: M C L 1$ ratios. In this trial, $38 \%$ of the patients with $t(11 ; 14)$ expressed a high $B C L 2: B C L 2 L 1$ gene expression ratio, while only $5 \%$ of the patients without $t(11 ; 14)$ had a high ratio of the gene expression [2,5,9]. Interestingly, KMS12PE and KMS27, which demonstrated high BCL-2:BCL-XL and BCL-2:MCL-1 ratios, were sensitive to venetoclax; in contrast, U266 and KM5, which showed low ratios, were resistant to venetoclax. The results of our in vitro experiments are consistent with the results of this clinical trial.

Our data correlated with those from a previous case report [13] and with the currently reported results of phase I/II trials for patients with MM [14,15]. A case report of a patient with relapsed plasma cell leukemia and $t(11 ; 14)$ treated with a combination therapy of venetoclax, daratumumab, and dexamethasone showed a rapid decrease in tumor burden [13]. Another group reported a phase I/II trial comprising 48 patients with R/R $\mathrm{MM}$ that were treated with a combination therapy of venetoclax and daratumumab to evaluate the efficacy and safety of this treatment strategy. Twenty-four MM patients with $t(11 ; 14)$ who were treated with more than one prior line of therapy (including PIs or IMiDs) were administered the combination therapy VenDd. Among these patients, $46 \%$ were refractory to PI, $71 \%$ were refractory to IMiDs, and $42 \%$ were double refractory to PI and IMiDs. The ORR of patients with $t(11 ; 14)$ treated by VenDd therapy was $92 \%$, while that for patients treated with venetoclax (Ven) in combination with daratumumab (D), dexamethasone $(\mathrm{d})$, and bortezomib $(\mathrm{V})(\mathrm{VenDVd})$ therapy was $88 \%$; this demonstrated a significant efficacy of the combination treatment $[14,15]$. Our data are also useful for establishing the optimal dose for each of the drugs. The effective treatment concentration can be evaluated based on in vitro data using samples obtained from patients.

Our data strongly suggested that natural killer (NK) cells functioned in vitro. Although the efficacy of the combination therapy of venetoclax and daratumumab has already been shown in clinical trials, our data provide significant meaning with the presence of ADCC activity by NK cells in vitro.

The induction of apoptosis by NK cells is one of the mechanisms of action of daratumumab [16]. In vitro data demonstrated that the level of CD38 expression in MM cells is correlated with the ADCC induction by daratumumab [17], and CD38 is produced in both NK and MM cells [18]. In light of these results, we need to further evaluate whether the level of CD38 expression in MM cells affects treatment response after the combination therapy of venetoclax and daratumumab. This should be performed using in vitro assays with samples taken from patients. The number of NK cells rapidly decreased in both the bone marrow and peripheral blood after daratumumab monotherapy [18]. This reduction in NK cells results in cytotoxicity among neighboring NK cells (NK cell fratricide) [19]. NK cells with low or no CD38 expression were resistant to their own cytotoxicity induced by daratumumab in an experiment using the peripheral blood and bone marrow of MM patients or healthy donors [19]. Indeed, these cells demonstrated superior ADCC activity induced by daratumumab than NK cells presenting higher CD38 expression levels $[19,20]$. Whether CD38 expression is correlated with the therapeutic response to a combination therapy of venetoclax and daratumumab remains to be evaluated.

This study has some limitations. It has been reported that the antimyeloma effect of daratumumab is induced by complement-dependent cytotoxicity (CDC) and antibody- 
dependent cellular phagocytosis (ADCP), in addition to ADCC as shown in this study [21-23]. Therefore, we attempted additional experiments to confirm whether venetoclax could enhance CDC by daratumumab using two CD38-positive myeloma cell lines (KMS12PE [10] and KMS27 [11]), which were used in this study and had revealed daratumumab mediated ADCC. However, daratumumab did not cause CDC in either of the two myeloma cell lines before examining the CDC-enhancing effect of venetoclax, as shown in Supplementary Figure S1. These results may be supported by a previous report [16] that analysis of tumor cells in patients with myeloma found only half of the patients had daratumumabinduced CDC. Therefore, it remains unclear whether CDC and ADCP could be involved in the synergistic effect of daratumumab and venetoclax on myeloma cells. However, venetoclax specifically inhibits BCL-2 in mitochondrial, causing caspase activation via the mitochondrial apoptotic pathway and exerting cytotoxic activity [24-26]. In ADCC by NK cells, the intracellular entry of granzyme through perforin pore formation induces caspase activation via the mitochondrial apoptotic pathway [27]. These suggest that the synergistic effect of ADCC and venetoclax by NK cells via daratumumab on MM cells may have been induced by activation of the mitochondrial apoptotic pathway. In contrast, the antitumor effects of CDC and ADCP are caused by the direct cell-killing effect by lysis through complement pore formation and phagocytosis by macrophages, respectively, and it is considered that the mitochondrial apoptosis pathway is not directly involved [28-31]. These suggest that venetoclax is unlikely to enhance CDC or ADCP against MM cells via daratumumab. However, these are highly speculative and require further investigation in the future.

In the future, both the characterization of MM samples and the measurement of NK cell activity should be essential aspects for determining a treatment strategy for MM. Analyzing the expression of proteins from the BCL-2 family in MM cells of the patient and predicting the treatment response may be useful for therapeutic decision-making. BH3 profiling is an example of a functional assay that investigates the interactions of BCL-2 family members [32]. These genetic and/or functional assays are useful tools for detecting the initial therapeutic target for the newly diagnosed patient by identifying the pro-survival proteins the tumor cells depend on [4]. Enhancing the therapeutic response by measuring and increasing NK cell activity is a promising treatment strategy, and several protocols for the expansion of NK cell activity are currently being investigated [33].

\section{Materials and Methods}

\subsection{Drugs and Cell Lines}

Venetoclax was purchased from Selleck (Houston, TX, USA). Daratumumab was purchased from Janssen (South Raritan, NJ, USA). The MM cell lines KMS12PE [10], KMS27 [11], U266 [34] and KM5 [35] were preserved at the laboratory of the Division of Hematology, Department of Internal Medicine of Aichi Medical University, in Nagakute, Japan. Cell lines were cultured in Roswell Park Memorial Institute (RPMI)-1640 medium with L-glutamine and sodium bicarbonate (R8758-500ML, Sigma-Aldrich, St. Louis, MO, USA), 10\% fetal bovine serum (Thermo Fisher Scientific, Waltham, MA, USA), and 1\% penicillin-streptomycin (Thermo Fisher Scientific, Waltham, MA, USA).

\subsection{Flow Cytometry}

MM cell lines were stained using an Alexa Fluor 647 Mouse Anti-human CD38 antibody (BioLegend, San Diego, CA, USA) for CD38 detection and an Alexa Fluor 647 Mouse IgG2a, $\mathrm{k}$ Isotype Ctrl antibody (BioLegend, San Diego, CA, USA) as a control. The stained cells were analyzed using a BD Fortessa cytometer (BD Biosciences, San Jose, CA, USA). Data were analyzed using the FlowJo software package (v. 10; Tree Star, Ashland, OR, USA).

\subsection{Western Blotting}

Aliquots of $1 \times 10^{6}$ cells were lysed in $20 \mu \mathrm{L}$ of ice-cold protein extraction buffer (10 mM Tris- $\mathrm{HCl}, 150 \mathrm{mM} \mathrm{NaCl}, 2 \mathrm{mM}$ ethylenediaminetetraacetic acid [EDTA], and $2 \mathrm{mM}$ 
2-mercaptoethanol [pH 7.4]) in 1.5-mL microtubes for $30 \mathrm{~min}$. The soluble fraction was separated by centrifugation at $10,000 \times g ; 15 \mu \mathrm{L}$ of the supernatant was mixed with $5 \mu \mathrm{L}$ of $4 \times$ sample buffer. The mixture was boiled for $3 \mathrm{~min}$ at $95^{\circ} \mathrm{C}$, and finally $5 \mu \mathrm{L}$ was loaded on a $4-12 \%$ sodium dodecyl sulfate (SDS)-polyacrylamide gradient gel (Novex NuPAGE SDS-PAGE, Invitrogen, Carlsbad, CA, USA). Proteins were fractionated by electrophoresis at $40 \mathrm{~mA}$ for $1 \mathrm{~h}$. The separated proteins were transferred to polyvinylidene fluoride (PVDF) membranes using an iBlot 2 Dry Blotting System (Invitrogen, Carlsbad, CA, USA). The membrane was incubated with $5 \%$ skim milk in phosphate-buffered saline (PBS) for $30 \mathrm{~min}$ at room temperature and then with $1 \mu \mathrm{g} / \mathrm{mL}$ of anti-BCL-2 antibody (MBL, Nagoya, Japan), $1 \mu \mathrm{g} / \mathrm{mL}$ of anti-BCL-XL antibody (MBL, Nagoya, Japan), $1 \mu \mathrm{g} / \mathrm{mL}$ of anti-MCL-1 antibody (CST, Danvers, MA, USA), or $0.1 \mu \mathrm{g} / \mathrm{mL}$ of anti- $\beta$-actin antibody (MBL, Nagoya, Japan) with $1 \%$ skim milk in PBS for $1 \mathrm{~h}$ at room temperature. After washing six times with PBS for $5 \mathrm{~min}$, the membrane was incubated with horseradish peroxidase-conjugated anti-mouse IgG (ImmPRESS HRP REAGENT PEROXIDASE Anti-Mouse IgG, Vector Laboratories, Burlingame, CA, USA) for $30 \mathrm{~min}$ at room temperature and washed again. The membrane was developed using a chemical luminescence system. Specific signals were detected with an Image Analyzer (Amersham, GE Healthcare, Chicago, IL, USA).

\subsection{WST-1 Assay}

MM cell lines were resuspended with culture medium at a concentration of $5 \times$ $10^{5} \mathrm{cel}-\mathrm{ls} / \mathrm{mL}$. Cell suspensions $(100 \mu \mathrm{L})$ were seeded in a 96-well flat-bottom plate and cultured at $37^{\circ} \mathrm{C}$ for $48 \mathrm{~h}$ in the presence of serial concentrations of venetoclax. In each well, $10 \mu \mathrm{L}$ of the Cell Counting Kit reagent (CytoSelect WST-1 Cell Proliferation Assay Reagent [Colorimetric], CBL, San Diego, USA) was added and the color reaction was performed at $37^{\circ} \mathrm{C}$ for $2 \mathrm{~h}$. Absorbance values were determined using a microplate reader (BIO-RAD, Hercules, CA, USA) at 450 and $620 \mathrm{~nm}$. We calculated cell viability using the formula as follows:

$$
\text { Cell viability }(\%)=\frac{\text { absorbance of the sample }- \text { blank }}{\text { absorbance of the negative control }- \text { blank }} \times 100
$$

The $x$-axis (on a base 10 logarithmic scale) represents venetoclax concentration and the $y$-axis shows the percentage of cell viability. The sample wells included cell lines and Cell Counting Kit reagent with venetoclax at the indicated concentrations, while the negative control included the cell lines and Cell Counting Kit reagent without venetoclax. The blank included the medium and Cell Counting Kit reagent without cells.

\subsection{NK Cell Preparation}

Peripheral blood mononuclear cells (PBMCs) obtained from a healthy donor were separated by a density gradient at $1500 \mathrm{rpm}$ for $30 \mathrm{~min}$ using Ficoll-Paque PLUS (GE Healthcare, Chicago, IL, USA). NK cells were separated using anti-CD56 antibody-coated magnetic micro beads in an autoMACS Pro Separator (Miltenyi Biotec, Bergisch Gladbach, Germany) and expanded using an NK Cell Activation/Expansion Kit (Miltenyi Biotec, Bergisch Gladbach, Germany) according to the manufacturer's protocol.

\subsection{Annexin $V$ Assay}

The MM cell lines KMS12PE, KMS27, U266, and KM5 were used as target cells for NK cell-mediated ADCC activity assessment using an annexin $\mathrm{V}$ assay. NK cells were co-incubated with KMS12PE or KM5 at a 0.3:1 effector: target ratio (E:T) for $18 \mathrm{~h}$ in the presence of serial concentrations of daratumumab and venetoclax and were analyzed by flow cytometry. These cells were stained with anti-CD56-PE (BioLegend, San Diego, CA, USA), a specific cell surface marker for NK cells and annexin V (BioLegend, San Diego, CA, USA), which is specific for apoptosis. The stained cells were analyzed with a BD Fortessa cytometer (BD Biosciences, San Jose, CA, USA) and data were analyzed using the FlowJo software package (v. 10; Tree Star, Ashland, OR, USA). Cytotoxicity was calculated as 
follows: cytotoxicity $(\%)=[($ the measured value at each daratumumab concentration with or without venetoclax) - (the measured value when no daratumumab or venetoclax was used)]/[100 — (the measured value when no daratumumab or venetoclax was used) $\times 100]$. The $x$-axis represents the concentrations of daratumumab and venetoclax whereas the $\mathrm{y}$-axis shows the cytotoxicity percentage.

\subsection{Perforin Inhibition by $C M A$}

CMA was used as a perforin inhibitor to investigate the downstream effector mechanisms of the combination treatment of daratumumab and venetoclax. KMS12PE cells were cocultured with NK cells, daratumumab, and venetoclax in the presence of $10 \mathrm{nM} \mathrm{CMA}$ for $18 \mathrm{~h}$, and cytotoxicity was analyzed by flow cytometry with annexin $\mathrm{V}$ following the method described in 4.6.

\subsection{Statistical Analysis}

Differences between the two groups were examined via the Student's t-test.

Supplementary Materials: The following are available online at https:/ /www.mdpi.com/article/10 $.3390 / \mathrm{ijms} 221910761 / \mathrm{s} 1$.

Author Contributions: Conceptualization, A.N., S.S., I.H., M.S. and A.T.; Formal Analysis, S.S.; Funding Acquisition, S.S. and I.H.; Investigation, A.N. and S.S.; Methodology, S.S.; Project Administration, S.S.; Resources, J.K., M.E. and I.H.; Supervision, R.U. and A.T.; Validation, S.S.; Writing-Original Draft, A.N.; Writing — Review and Editing, S.S., M.S. and A.T. All authors have read and agreed to the published version of the manuscript.

Funding: This research was funded by grants-in-aid for scientific research (grant number 18K07277 to Susumu Suzuki, grant number 19K08825 to Ichiro Hanamura and 21K08427 to Akiyoshi Takami).

Institutional Review Board Statement: The study was conducted according to the guidelines of the Declaration of Helsinki and approved by the Institutional Review Board of Aichi Medical University (protocol code: 2020-H073 and date of approval: 4 November 2020).

Informed Consent Statement: Informed consent was obtained from all subjects involved in the study.

Data Availability Statement: Not applicable.

Acknowledgments: We thank Hiromi Tsuchida for excellent technical assistance, and Kyoko Okumura and Yuka Oohigashi for excellent secretarial assistance. We thank Makoto Naruse and Natsumi Kodama (Aichi Medical University, Institute of Comprehensive Medical Research, Division of Advanced Research Promotion) for the maintenance of the BD LSRFortessa flow cytometer and Amersham Imager 600.

Conflicts of Interest: Ichiro Hanamura received research funding from Bristol-Myers Squibb, MSD K.K., Ono Pharmaceutical, Kyowa Kirin, Sanofi K.K., Taiho Pharmaceutical, Takeda Pharmaceutical, Chugai Pharmaceutical, Novartis AG, and Celgene K.K., and received honoraria from Celgene K.K., Janssen, Takeda Pharmaceutical, Ono Pharmaceutical, Bristol-Myers Squibb, Novartis AG, and AbbVie GK. Ryuzo Ueda received research funding from Kyowa Kirin, Chugai Pharmaceutical, and Ono Pharmaceutical. The other authors have no conflict of interest to disclose.

\section{References}

1. Rajkumar, S.V. Multiple myeloma: 2020 update on diagnosis, risk-stratification and management. Am. J. Hematol. 2020, 95, 548-567. [CrossRef] [PubMed]

2. Touzeau, C.; Maciag, P.; Amiot, M.; Moreau, P. Targeting Bcl-2 for the treatment of multiple myeloma. Leukemia 2018, 32, 1899-1907. [CrossRef] [PubMed]

3. Touzeau, C.; Dousset, C.; Le Gouill, S.; Sampath, D.; Leverson, J.D.; Souers, A.J.; Maïga, S.; Béné, M.C.; Moreau, P.; Deceunynck, C.; et al. The Bcl-2 specific BH3 mimetic ABT-199: A promising targeted therapy for $\mathrm{t}(11 ; 14)$ multiple myeloma. Leukemia 2013, 28, 210-212. [CrossRef] [PubMed]

4. Valentin, R.; Grabow, S.; Davids, M.S. The rise of apoptosis: Targeting apoptosis in hematologic malignancies. Blood 2018, 132, 1248-1264. [CrossRef] [PubMed] 
5. Kumar, S.; Kaufman, J.L.; Gasparetto, C.; Mikhael, J.; Vij, R.; Pegourie, B.; Benboubker, L.; Facon, T.; Amiot, M.; Moreau, P.; et al. Efficacy of venetoclax as targeted therapy for relapsed/refractory $t(11 ; 14)$ multiple myeloma. Blood 2017, 130, 2401-2409. [CrossRef] [PubMed]

6. Moreau, P.; Chanan-Khan, A.; Roberts, A.; Agarwal, A.B.; Facon, T.; Kumar, S.; Touzeau, C.; Punnoose, E.A.; Cordero, J.; Munasinghe, W.; et al. Promising efficacy and acceptable safety of venetoclax plus bortezomib and dexamethasone in relapsed/refractory MM. Blood 2017, 130, 2392-2400. [CrossRef]

7. Gomez-Bougie, P.; Wuillème-Toumi, S.; Ménoret, E.; Trichet, V.; Robillard, N.; Philippe, M.; Bataille, R.; Amiot, M. Noxa Upregulation and Mcl-1 Cleavage Are Associated to Apoptosis Induction by Bortezomib in Multiple Myeloma. Cancer Res. 2007, 67, 5418-5424. [CrossRef] [PubMed]

8. Punnoose, E.A.; Leverson, J.D.; Peale, F.; Boghaert, E.R.; Belmont, L.D.; Tan, N.; Young, A.; Mitten, M.; Ingalla, E.; Darbonne, W.C.; et al. Expression Profile of BCL-2, BCL-XL, and MCL-1 Predicts Pharmacological Response to the BCL-2 Selective Antagonist Venetoclax in Multiple Myeloma Models. Mol. Cancer Ther. 2016, 15, 1132-1144. [CrossRef] [PubMed]

9. Matulis, S.M.; Gupta, V.; Nooka, A.K.; Von Hollen, H.; Kaufman, J.L.; Lonial, S.; Boise, L.H. Dexamethasone treatment promotes Bcl-2 dependence in multiple myeloma resulting in sensitivity to venetoclax. Leukemia 2015, 30, 1086-1093. [CrossRef]

10. Namba, M.; Ohtsuki, T.; Mori, M.; Togawa, A.; Wada, H.; Sugihara, T.; Yawata, Y.; Kimoto, T. Establishment of five human myeloma cell lines. In Vitro Cell. Dev. Biol. 1989, 25, 723-729. [CrossRef]

11. Bose, P.; Gandhi, V.V.; Konopleva, M.Y. Pathways and mechanisms of venetoclax resistance. Leuk. Lymphoma 2017, 58, 2026-2039. [CrossRef]

12. Offidani, M.; Corvatta, L.; Morè, S.; Nappi, D.; Martinelli, G.; Olivieri, A.; Cerchione, C. Daratumumab for the Management of Newly Diagnosed and Relapsed/Refractory Multiple Myeloma: Current and Emerging Treatments. Front. Oncol. 2021, 10. [CrossRef]

13. D'Agostino, M.; Innorcia, S.; Boccadoro, M.; Bringhen, S. Monoclonal Antibodies to Treat Multiple Myeloma: A Dream Come True. Int. J. Mol. Sci. 2020, 21, 8192. [CrossRef]

14. Roccatello, D.; Fenoglio, R.; Sciascia, S.; Naretto, C.; Rossi, D.; Ferro, M.; Barreca, A.; Malavasi, F.; Baldovino, S. CD38 and Anti-CD38 Monoclonal Antibodies in AL Amyloidosis: Targeting Plasma Cells and beyond. Int. J. Mol. Sci. 2020, 21, 4129. [CrossRef]

15. Otsuki, T.; Sakaguchi, H.; Hatayama, T.; Wu, P.; Takata, A.; Hyodoh, F. Effects of All-transRetinoic Acid (ATRA) on Human Myeloma Cells. Leuk. Lymphoma 2003, 44, 1651-1656. [CrossRef]

16. De Weers, M.; Tai, Y.-T.; Van Der Veer, M.S.; Bakker, J.M.; Vink, T.; Jacobs, D.C.H.; Oomen, L.A.; Peipp, M.; Valerius, T.; Slootstra, J.W.; et al. Daratumumab, a Novel Therapeutic Human CD38 Monoclonal Antibody, Induces Killing of Multiple Myeloma and Other Hematological Tumors. J. Immunol. 2010, 186, 1840-1848. [CrossRef]

17. Pan, R.; Hogdal, L.J.; Benito, J.M.; Bucci, D.; Han, L.; Borthakur, G.; Cortes, J.; DeAngelo, D.J.; DeBose, L.; Mu, H.; et al. Selective BCL-2 Inhibition by ABT-199 Causes On-Target Cell Death in Acute Myeloid Leukemia. Cancer Discov. 2013, 4, 362-375. [CrossRef]

18. Joza, N.; Susin, S.A.; Daugas, E.; Stanford, W.L.; Cho, S.K.; Li, C.Y.J.; Sasaki, T.; Elia, A.J.; Cheng, H.-Y.M.; Ravagnan, L.; et al. Essential role of the mitochondrial apoptosis-inducing factor in programmed cell death. Nature 2001, 410, 549-554. [CrossRef] [PubMed]

19. Di Martino, L.; Tosello, V.; Peroni, E.; Piovan, E. Insights on Metabolic Reprogramming and Its Therapeutic Potential in Acute Leukemia. Int. J. Mol. Sci. 2021, 22, 8738. [CrossRef] [PubMed]

20. Martínez-Lostao, L.; Anel, A.; Pardo, J. How Do Cytotoxic Lymphocytes Kill Cancer Cells? Clin. Cancer Res. 2015, 21, 5047-5056. [CrossRef] [PubMed]

21. Golay, J.; Taylor, R. The Role of Complement in the Mechanism of Action of Therapeutic Anti-Cancer mAbs. Antibodies 2020, 9, 58. [CrossRef]

22. Gül, N.; van Egmond, M. Antibody-Dependent Phagocytosis of Tumor Cells by Macrophages: A Potent Effector Mechanism of Monoclonal Antibody Therapy of Cancer. Cancer Res. 2015, 75, 5008-5013. [CrossRef]

23. Young, J.D.; Ko, S.S.; Cohn, Z.A. The increase in intracellular free calcium associated with IgG gamma $2 \mathrm{~b} /$ gamma 1 Fc receptorligand interactions: Role in phagocytosis. Proc. Natl. Acad. Sci. USA 1984, 81, 5430-5434. [CrossRef] [PubMed]

24. Markiewski, M.M.; Lambris, J.D. Is complement good or bad for cancer patients? A new perspective on an old dilemma. Trends Immunol. 2009, 30, 286-292. [CrossRef]

25. Nalghranyan, S.; Singh, A.P.; Schinke, C. The combination of venetoclax, daratumumab and dexamethasone for the treatment of refractory primary plasma cell leukemia. Am. J. Hematol. 2019, 95, E34-E35. [CrossRef] [PubMed]

26. Bahlis, N.; Baz, R.; Harrison, S.; Quach, H.; Ho, S.-J.; Vangsted, A.; Moreau, P.; Gibbs, S.; Salem, A.; Ross, J.; et al. First Analysis from a Phase 1/2 Study of Venetoclax in Combination with Daratumumab and Dexamethasone, +/- Bortezomib, in Patients with Relapsed/Refractory Multiple Myeloma. Blood 2019, 134, 925. [CrossRef]

27. Terpos, E.; Ntanasis-Stathopoulos, I. Clinical Updates Regarding Multiple Myeloma from the 2019 American Society of Hematology Annual Meeting. Clin. Lymphoma Myeloma Leuk. 2020, 20, 499-508. [CrossRef] [PubMed]

28. Nijhof, I.S.; Groen, R.; Lokhorst, H.M.; Van Kessel, B.; Bloem, A.C.; Van Velzen, J.; De Jong-Korlaar, R.; Yuan, H.; Noort, W.A.; Klein, S.K.; et al. Upregulation of CD38 expression on multiple myeloma cells by all-trans retinoic acid improves the efficacy of daratumumab. Leukemia 2015, 29, 2039-2049. [CrossRef] 
29. Casneuf, T.; Xu, X.S.; Adams, H.C., 3rd; Axel, A.E.; Chiu, C.; Khan, I.; Ahmadi, T.; Yan, X.; Lonial, S.; Plesner, T.; et al. Effects of daratumumab on natural killer cells and impact on clinical outcomes in relapsed or refractory multiple myeloma. Blood Adv. 2017, 1, 2105-2114. [CrossRef] [PubMed]

30. Wang, Y.; Zhang, Y.; Hughes, T.; Zhang, J.; Caligiuri, M.A.; Benson, D.M.; Yu, J. Fratricide of NK Cells in Daratumumab Therapy for Multiple Myeloma Overcome by Ex Vivo-Expanded Autologous NK Cells. Clin. Cancer Res. 2018, 24, 4006-4017. [CrossRef]

31. Krejcik, J.; Frerichs, K.A.; Nijhof, I.S.; Van Kessel, B.; Van Velzen, J.F.; Bloem, A.C.; Broekmans, M.E.; Zweegman, S.; Van Meerloo, J.; Musters, R.J.; et al. Monocytes and Granulocytes Reduce CD38 Expression Levels on Myeloma Cells in Patients Treated with Daratumumab. Clin. Cancer Res. 2017, 23, 7498-7511. [CrossRef]

32. Certo, M.; Moore, V.D.G.; Nishino, M.; Wei, G.; Korsmeyer, S.; Armstrong, S.A.; Letai, A. Mitochondria primed by death signals determine cellular addiction to antiapoptotic BCL-2 family members. Cancer Cell 2006, 9, 351-365. [CrossRef] [PubMed]

33. Granzin, M.; Wagner, J.; Köhl, U.; Cerwenka, A.; Huppert, V.; Ullrich, E. Shaping of Natural Killer Cell Antitumor Activity by Ex Vivo Cultivation. Front. Immunol. 2017, 8, 458. [CrossRef] [PubMed]

34. Nilsson, K.; Bennich, H.; Johansson, S.G.O.; Pontén, J. Established immunoglobulin producing myeloma (IgE) and lymphoblastoid (IgG) cell lines from an IgE myeloma patient. Clin. Exp. Immunol. 1970, 7, 477-489.

35. Hanamura, I.; Iida, S.; Akano, Y.; Hayami, Y.; Kato, M.; Miura, K.; Harada, S.; Banno, S.; Wakita, A.; Kiyoi, H.; et al. Ectopic expression of MAFB gene in human myeloma cells carrying (14;20)(q32;q11) chromosomal translocations. Jpn. J. Cancer Res. 2001, 92, 638-644. [CrossRef] [PubMed] 